\title{
Pro-oncogene Pokemon Promotes Prostate Cancer Progression by Inducing STRN4 Expression
}

\author{
Fuquan Jiang1, Qingfan Zheng1, Liping Chang², Xu Li1, Xinsheng Wang ${ }^{3 凶}$ and Xinquan $\mathrm{Gu}^{1 \bowtie}$ \\ 1. Department of Urology, China-Japan Union Hospital of Jilin University, Jilin, China \\ 2. The First Affiliated Hospital, Changchun University of Chinese Traditional Medicine, Jilin, China \\ 3. Department of Urology, Tianjin First Center Hospital, Tianjin, China \\ $\square$ Corresponding authors: Xinsheng Wang: wxsdoctor@126.com; Department of Urology, Tianjin First Center Hospital, Tianjin, 300192 China. Tel./Fax: \\ +86-02223626850 Or Xinquan Gu : guxqprofessor@163.com; Department of Urology, China-Japan Union Hospital of Jilin University, Jilin, 130033 China. \\ Tel./Fax: +86-043184995141 \\ (1) Ivyspring International Publisher. This is an open access article distributed under the terms of the Creative Commons Attribution (CC BY-NC) license \\ (https://creativecommons.org/licenses/by-nc/4.0/). See http://ivyspring.com/terms for full terms and conditions.
}

Received: 2018.08.25; Accepted: 2019.03.04; Published: 2019.04.21

\begin{abstract}
Pokemon, also known as leukemia/lymphoma-related factor (LRF) is a pro-oncogenic protein highly expressed in several cancers. There have been few in vitro and animal studies about its malignant biological behavior and function, however, its role especially in prostate cancer has not been completely elucidated. Therefore, in this study, we identified that Pokemon is overexpressed in human prostate cancer tissue samples, and its suppression inhibits proliferation of prostate cancer cells, along with promotion of apoptosis. Furthermore, to explore the mechanism by which Pokemon promotes tumor progression, we observed that it binds to the promoter of STRN4 (striatin 4), a downstream target, and subsequently regulates its expression. In conclusion, our study indicated that Pokemon through stimulation of STRN4 expression promotes prostate tumor progression via a Pokemon /STRN4 axis.
\end{abstract}

Key words: Pokemon, STRN4, prostate cancer, proliferation, apoptosis

\section{Introduction}

Pokemon, also known as leukemia/lymphomarelated factor (LRF) is a member of POK family of transcriptional repressors [1], and seems to have pro-oncogenic role along with high expression in several cancers including non-small lung cancer, $\mathrm{T}$ and B-cell lymphomas, ovarian carcinomas, gliomas, prostate and breast cancer[2-5]. Earlier studies have indicated that pokemon promote cell transformation by inhibiting tumor suppressor ARF/p53 pathway [1, 6]. In addition, it has also been shown to interfere with GC box recognition by Sp1, and repress ADH5/FDH transcription [7, 8]. Other studies have demonstrated that it also activates membrane type- 1 matrix metalloproteinase in ovarian cancer, p14ARF in lymphomas, cyclin $\mathrm{A}$ in adipogenesis, and affects the transcription of nuclear factor (NF)-KB-activated genes by interacting with NF-kB p65 subunit[9-11]. Despite these in vitro studies along with animal experiments, its malignant biological behavior and function in prostate cancer is still not fully explored.

STRN4, a member of the striatin family of proteins is overexpressed in the central and peripheral nervous systems [12]. These proteins contain various protein-binding domains including coiled-coil domain, $\mathrm{Ca}^{2+}$-calmodulin-binding domain, caveolin-binding domain and WD-repeat domain [13, 14]. These domains typically promotes dimerization of striatin family members, along with their interaction with several other proteins, like calmodulin, protein phosphatase 2A (PP2A), caveolin and diverse set of protein kinases. Previously studies have reported that STRN4 can associate with several members of the germinal center kinase family, such as misshapen-like kinase 1 (MINK1), mitogen-activated protein kinase kinase kinase kinase 4 (MAP4K4), Traf2- and NCK-interacting protein (TNIK). Interestingly all these proteins are involved in either tumor suppression or progression. In addition, 
STRN4 knockdown has been shown to suppress proliferation, invasion and migration of multiple cancer cell types, including lung, esophageal, gastric, pancreatic, ovarian, breast and colorectal, thereby functionally implicating STRN4 in cancer progression[15]. However, the link between STRN4 and prostate tumor progression is still missing.

In this study, we identified that Pokemon was overexpressed in human prostate cancer tissues, and its suppression inhibited prostate cancer cell proliferation but promoted their apoptosis. Moreover, we also demonstrated that STRN4 is a downstream target of Pokemon, and their expression highly correlates in human prostate cancer. Further, our study revealed that Pokemon through direct binding to STRN4 promoter regulates its expression, which in turn might promote prostate tumor progression.

\section{Materials and Methods}

\section{Patient samples}

We collected 126 prostate cancer tissue samples between the year 2012 and 2015, after surgical resection from China-Japan Union Hospital of Jilin University (Jilin, China). None of the patient had any anticancer therapy before tissue sample collection. The tumor stage was characterized using guidelines from 2010 American Joint Committee on Cancer and International Union against Cancer tumor-nodemetastasis (TNM) classification system, while the tumor differentiation was graded according to the Edmondson and Steiner grading system. This study was approved by the Ethics Committee of the China-Japan Union Hospital of Jilin University (Jilin, China), and all patients provided written informed consent. The clinicopathological characteristics of these patients have been shown in Table 1.

\section{Immunohistochemical staining}

The surgical tissue specimens were first embedded in paraffin blocks, and then sliced into 6 $\mu \mathrm{m}$ thick sections. Next, after antigen retrieval and blocking, anti-human Pokemon or STRN4 (Abcam) antibodies were used for immunohistochemical staining, as described previously (Wei, Wu et al. 2006). After staining, the images were captured under a light microscope (Zeiss, Germany). Finally, based on the pokemon or STRN4 staining intensity, the patients were classified into high $(+)$ and low (-) expression groups.

\section{Cell culturing}

The PC3 and DU145 human prostate cancer cell lines were procured from ATCC (American Type Cell Collection, Manassas, VA, USA), and both cell types were cultured in complete DMEM medium supplemented with 10\% FBS (Gibco, USA), 100 units $/ \mathrm{ml}$ streptomycin, and 100 units/ml penicillin (Invitrogen, Carlsbad, CA, USA) in an incubator at $37^{\circ} \mathrm{C}$ with $5 \% \mathrm{CO}_{2}$ atmosphere.

\section{Stable gene knockdown}

To generate stable knockdown of Pokemon and STRN4 in PC3 and DU145 cells, lentivirus mediated shRNA infection was performed. To make the lentivirus, HEK 293T cells were first transfected with STRN4 and Pokemon specific shRNAs in the pLKO.1-puro vector, along with GAG-pol, REV and VSVG genes expression vectors. After $48 \mathrm{hr}$ of transfection, lentivirus particles were harvested, and subsequently used to infect PC 3 and DU145 cells. Post $24 \mathrm{hr}$ of lentivirus infection, these cells were further

Table 1. Correlations between Pokemon expression and clinicopathological features in prostate cancer patients

\begin{tabular}{|c|c|c|c|c|}
\hline Features & Number & Pokemon e & expression & P-value \\
\hline & & Low & High & \\
\hline Age at Su & & & & \\
\hline$<60$ & 55 & 19 & 36 & 0.778 \\
\hline$\geqslant 60$ & 71 & 24 & 47 & \\
\hline Gleason s & & & & \\
\hline$<7$ & 96 & 65 & 31 & 0.032 \\
\hline$\geqslant 7$ & 30 & 17 & 13 & \\
\hline PSA level. & & & & \\
\hline$<10 \mathrm{ng} / \mathrm{mL}$ & 77 & 51 & 26 & 0.148 \\
\hline$\geqslant 10 \mathrm{ng} / \mathrm{ml}$ & 49 & 28 & 21 & \\
\hline Pathologi & & & & \\
\hline pT $2 a-c$ & 83 & 67 & 16 & 0.007 \\
\hline pT3a-4 & 43 & 20 & 23 & \\
\hline Lymph Nod & & & & \\
\hline Negative & 107 & 66 & 41 & 0.153 \\
\hline Positive & 19 & 11 & 8 & \\
\hline
\end{tabular}


treated with $1 \mu \mathrm{g} / \mathrm{mL}$ of puromycin (Sigma-Aldrich) to select stably infected cells. Two different shRNA sequences were used for stable knockdown of each of the genes, and their sequences are as follows, Pokemon shRNA1: CCGGATCCGCACTTTAAGGAC GAGGACTCTCGAGAGTCCTCGTCCTTAAAGTGC TTTTTTG. Pokemon shRNA2: CCGGGCCACTGAG ACACAAACCTATCTCGAGATAGGTTTGTGTCTC AGTGGCTTTTTTG. STRN4 shRNA1: CCGGACGAC TGTTCTCTGCGTTTATCTCGAGATAAACGCAGA GAACAGTCGTTTTTTG, STRN4 shRNA2: CCGGCC TGGACAATCGAACAGGTAACTCGAGTTACCTGT TCGATTGTCCAGGTTTTTG.

\section{Apoptotic assay}

The apoptotic activity was assessed using previously described method [16]. Briefly, PC3 and DU145 cells were harvested and centrifuged at $400 \times \mathrm{g}$ for $5 \mathrm{~min}$. Next, after discarding supernatant, the cell pellet was resuspended in $0.5 \mathrm{ml}$ of binding buffer. The suspended cells were further stained using Annexin V-FITC (BD Biosciences) and PI (propidium iodide) (Sigma Chemical) stains, and were analyzed by flow cytometry to identify apoptotic cells. Finally, the apoptosis rate was calculated using FlowJo 8.7.1 software.

\section{Cell cycle analysis}

For cell cycle analysis, PC3 and Du145 cells, were first resuspended in pre-cooled $70 \%$ alcohol, and later suspended as single-cell suspension. The fixed and suspended cells were then centrifuged and washed 3 times with 1X PBS. These cells were later incubated with RNase A $(1 \mathrm{mg} / \mathrm{mL})$ for $30 \mathrm{~min}$ at $37^{\circ} \mathrm{C}$, and then with PI solution ( $20 \mu \mathrm{g} / \mathrm{mL}$; Sigma Chemical) for $30 \mathrm{~min}$. The cell cycle analysis was subsequently performed using FACSCanto II flow cytometer and Flowjo 8.7.1 software (BD Bioscience).

\section{Cell proliferation}

To assess the cell proliferation, PC3 and DU145 cells were plated into 96-well plates (3000 cells/well), in triplicate. The proliferation at various time points was monitored using MTT cell proliferation reagent kit I (Roche, Basel, Switzerland) as per manufacturer's instructions. For each treatment group, the proliferation rate was assessed as average of the triplicate wells.

\section{Quantitative real-time PCR}

Total RNA from the cultured PC3 and DU145 cells was extracted using Trizol reagent (Invitrogen), according to the manufacturer's protocol. The isolated RNA was reverse transcribed using MLV reverse transcriptase (Invitrogen), and quantitative PCR analysis was performed with SYBR Premix Ex Taq
(Takara) reagent, as per manufacturer's instructions. The target gene expression was normalized to housekeeping gene, Glyceraldehyde 3-phosphate dehydrogenase (GAPDH). The primers used in PCR reaction were synthesized by Sangon Biotech (China), and their sequences are as follows, Human Pokemon Forward: ACGAGTGCAACATCTGCAAG, and Reverse: GTCGTAGTTGTGGGCAAAGG; Human STRN4 Forward: GATCTCACCGTCACCAACGA, and Reverse: GGAACGAATGCCGTCGTAGT; Human GAPDH Forward: AATCCCATCACCATCTTCCA, and Reverse: GGCGGAGATGATGACCCTT; Human CyclinD Forward: TGTCCTACTACCGCCTCACA, and Reverse: CTTGACTCCAGCAGGGCTTC; Human Bax Forward: GGGGAGCAGCCCAGAGG, and Reverse: TGCTCGATCCTGGATGAAACC.

\section{Western blotting}

The protein lysates from PC3 and Du145 cells were prepared by using a lysis buffer (supplied by Cell Signaling Technology, Danvers, USA) containing protease inhibitor (Sigma Chemical Company, St. Louis, USA). The proteins were purified after centrifugation. Equal amount of protein was separated using gel electrophoresis, and transferred to the membrane using previously described method [17]. The proteins on the membranes were blotted using specific primary antibodies against Pokemon (1:1000, Abcam), STRN4 (1:1000, Abcam), GAPDH (1:1000, CST), CyclinD (1:1000, Abcam), and Bax (1:1000, CST). Relevant anti-rabbit IgG-HRP (1:5000, CST), and Goat anti-mouse IgG-HRP (1:5000, Santa Cruz) secondary antibodies were used. Finally the protein expression signal was recorded using chemiluminescent reagent.

\section{Illumina transcriptome library preparation and sequencing}

Total RNA from each sample was extracted using RN38 EASY spin Plus RNA Kit (Aidlab Biotechnologies Co., Ltd., Beijing, China), to prepare RNA library for transcriptome sequencing. The extracted RNA was first treated with RNase-free DNase I (Takara Inc., Kyoto, Japan) for $45 \mathrm{~min}$ at $37^{\circ} \mathrm{C}$ to remove residual DNA. The quantity and quality of isolated RNA was assessed using gel electrophoresis and spectrophotometric analysis (Quawell Q5000, San Jose, CA, USA). In addition, RNA integrity was measured using RNA Nano 6000 assay Kit from Agilent Bioanalyzer 2100 system (Agilent Technologies, CA, USA). Furthermore, mRNA isolation, cDNA synthesis, addition of adapters, PCR amplification and RNA-Seq was performed by the staff at Beijing Biomarker Technologies (Beijing, China). Briefly, a total of $3 \mathrm{mg}$ RNA per sample was 
used as input material for sample preparations. Next, the mRNA-Seq libraries were constructed, using NEB Next Ultra RNA Library Prep Kit from Illumina (NEB, USA). The mRNA was purified using oligo (dT) magnetic beads, and then digested into fragments using NEB Next First Strand Synthesis Reaction Buffer (5X). The fragmented mRNA was subsequently used to synthesize first-strand cDNA by using reverse transcriptase, RNase $\mathrm{H}$ - and random hexamer primers. The second strand cDNA synthesize was achieved using DNA Polymerase I, RNase $\mathrm{H}$, and dNTPs. Next, the PCR products were purified using AMPure XP system (Beckman Coulter, Beverly, USA) to construct cDNA libraries, which were subsequently assessed by Agilent Bioanalyzer 2100 system, and sequenced on an Illumina HiSequation 4000 platform. Finally, the paired-end reads were generated.

\section{Luciferase reporter assays}

Luciferase reporter assays were performed as described previously [18]. Briefly, PC3 cells were plated into $24-w e l l$ plates at $70-80 \%$ confluency, one day prior to transfection. Next day, plated cells were transfected with plasmid DNAs, including reporter plasmid, using Lipofectamine 2000 (Invitrogen) transfection reagent and incubated for $24 \mathrm{hr}$ according to the manufacturer's recommendations. Later, fresh media was added to the transfected cells and 48 hours after transfection, the cells were lysed with passive lysis Buffer to analyze luciferase activity using Dual-Luciferase Assay system (Promega). pRL-SV40Renilla was used as an internal control for transfection efficiency.

\section{Xenograft growth assay}

To assess the effect of Pokemon and STRN4 on tumor xenograft growth, PC3 cell expressing control, Pokemon and STRN4 shRNAs were injected into the ventral regions of six female 6 weeks old BALB/C nude mice. These BALB/C nude mice were maintained according to the guidelines from administration of laboratory animal research, and Institutional Animal Care and Use Committee of the China-Japan Union Hospital of Jilin University, along with the Care and Use of Laboratory Animals protocol (National Institutes of Health). Tumor growth of the injected cells was monitored weekly by measuring tumor length (L) and width (W), using Vernier caliper. The tumor volumes $(\mathrm{V})$ were calculated using the formula: $\mathrm{V}=\mathrm{LW}^{2} / 2$.

\section{Statistics}

All statistical analyses were performed with SPSS 17.0 software (SPSS, Chicago, IL), and each experiment was independently repeated three times. The measurement values were expressed as mean \pm standard deviation. To test the significance of the data, student's $t$ test, Spearman's rank correlation and ANOVA analysis were used. The $p$ value of $<0.05$ represented statistical significance.

\section{Results}

\section{Pokemon expression is elevated in human prostate cancer tissues}

To examine if Pokemon has any functional role in prostate cancer progression, we first analyzed its expression in human prostate cancer and normal tissue samples, using immunohistochemical staining. Based on the staining intensity from 126 prostate cancer biopsy specimens, it appeared that 83 (65.8\%) of them had high Pokemon expression (Figure 1A), while all normal tissues displayed low expression (Table 1). The elevated protein expression was further confirmed by western blotting in the prostate cancer tissues (Figure 1B). In addition, we also compared Pokemon expression at mRNA level (Figure 1C), which also indicated elevated expression in prostate cancer tissues, in comparison to normal tissue samples. Overall, our data demonstrated that Pokemon is indeed overexpressed in human prostate cancer tissues.

\section{Pokemon ablation inhibits prostate cancer cell proliferation and promotes apoptosis}

Next, to understand the functional contribution of elevated pokemon expression in prostate cancer cells, we analyzed their cell proliferation, cell cycle and apoptosis. The Pokemon expression knockdown in PC3 and DU145 cells was achieved with two different specific short hairpin RNAs (shRNA) (Figure 2A). Cell proliferation analysis of pokemon knockdown cells showed reduced proliferation in both PC3 and DU145 cell types, in comparison to control knockdown, as seen in Figure 2B. Next, we also analyzed the effects of pokemon knockdown on cell cycle and apoptosis. As seen in Figure 2C and 2D, Pokemon knockdown in PC3 and DU145 cells, not only significantly inhibited cell cycle, but also induced apoptosis. These results indicated that both inhibition of cell cycle progression and induction of apoptosis, might contribute to reduced proliferation due to Pokemon knockdown in prostate cancer cells. 
A
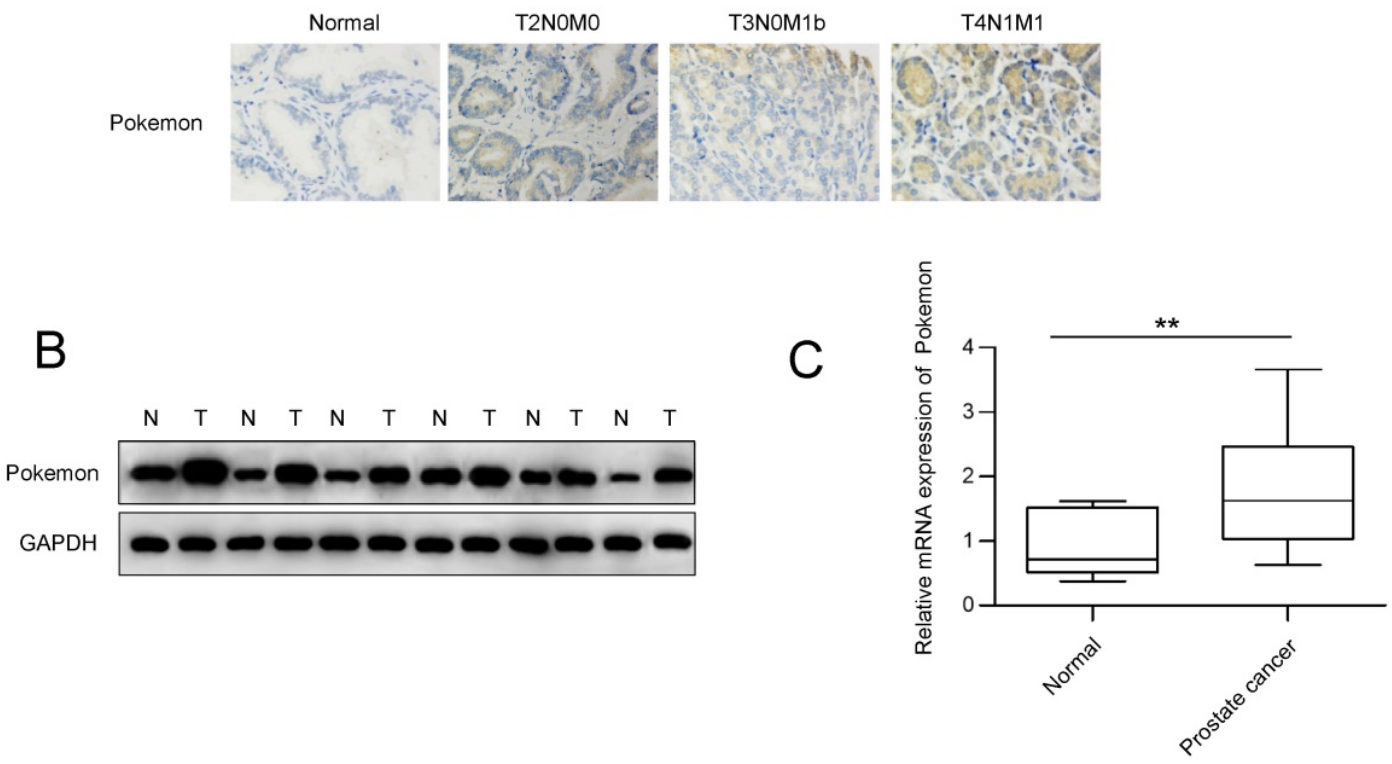

Figure 1. Analysis of Pokemon expression in human prostate cancer tissue specimens. (A) Immunohistochemistry analysis of Pokemon expression in normal and prostate cancer tissue samples. (B) Pokemon expression analysis in normal and prostate cancer tissue samples by western blotting. (C) $q R T P C R$ analysis of the Pokemon mRNA expression in normal and prostate cancer tissue samples. $* *$ p $<0.05$, mean \pm SEM.

\section{STRN4 is a downstream target of Pokemon}

To understand the molecular mechanism of Pokemon's role in promoting prostate cancer cell growth, we performed illumina transcriptome sequencing to identify genes regulated by it in PC3 cancer cells. Microarray analysis indicated more than two-fold change in expression of 121 genes after Pokemon knockdown in PC3 cells, in comparison to control knockdown (Figure 3A). Further analysis based on Kyoto Encyclopedia of Genes \& Genomes, and Gene Ontology databases, it was observed that majority of the Pokemon target genes were involved in signal transduction, biosynthesis metabolism, apoptosis, cell cycle and differentiation, thereby suggesting its role in proliferation and carcinogenesis of tumor cells (Figure 3B). Among the different pokemon target genes, we focused on STRN4, which was the top gene in cell cycle pathway, and possibly a tumor progression factor. The STRN4 protein expression was analyzed in prostate cancer cells (PC3 and DU145) having pokemon knockdown or overexpression. Interestingly, a concomitant expression pattern was observed for STRN4 and Pokemon (Figure 3C). These results indicated that Pokemon induced STRN4 expression in human prostate cancer cells.

\section{Pokemon and STRN4 expression correlates in human prostate cancer}

Next, we analyzed the expression of STRN4. qPCR analysis confirmed elevated mRNA expression of STRN4 in prostate cancer tissue samples, in comparison to normal tissue samples (Figure 4A). In addition, we also compared the expression correlation between pokemon and STRN4, by analyzing STRN4 expression through IHC analysis, in same 126 prostate cancer biopsy specimens that were earlier used for pokemon expression. Our analysis demonstrated that STRN4 was overexpressed in $77(61.1 \%)$ of the 126 prostate cancer tissues and showed high correlation with Pokemon expression, as seen in Figure 4B and C. This indicated a probable link between pokemon and STRN4, and it is possible that STRN4 may play a key role in promoting pokemon mediated prostate cancer progression.

\section{STRN4 promotes proliferation and inhibits apoptosis of prostate cancer cells}

We also tested directly the effects of STRN4 manipulation on prostate cancer cell growth, by knocking down its expression through specific shRNAs in PC3 and DU145 cells (Figure 5A). STRN4 knockdown clearly reduced the proliferation in both cell types (Figure 5B). Similarly, its knockdown also inhibited cell cycle progression and promoted apoptosis, as analyzed by Annexin V-FITC/PI staining (Figure 5C-D), in both PC3 and DU145 cell lines. Therefore, it appeared that STRN4 played a very similar role as pokemon, in impacting prostate cancer cell proliferation and apoptosis. 


\section{Pokemon and STRN4 cooperate to drive prostate cancer cells proliferation}

To further confirm a direct link between Pokemon and STRN4, we performed additional rescue experiments. The induction of cell cycle progression and inhibition of apoptosis by ectopic expression of Pokemon were effectively reversed after STRN4 knockdown in the same cells, as seen in Figure 6A-C. Additionally, in the cells with pokemon knock- down, the ectopic expression of STRN4 reversed the phenotype, by promoting cell proliferation. The similar results were also obtained for cell cycle and apoptosis-related markers, as analyzed by western blotting in prostate cancer cells (Figure 6D). These results clearly indicated that STRN4 is indeed a downstream target of pokemon, and they both cooperate to drive prostate cancer cells proliferation.

A

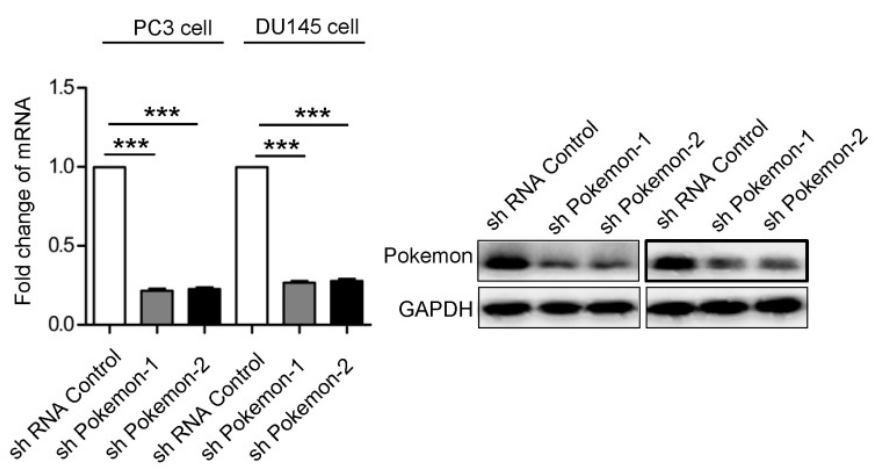

B

PC3 cell
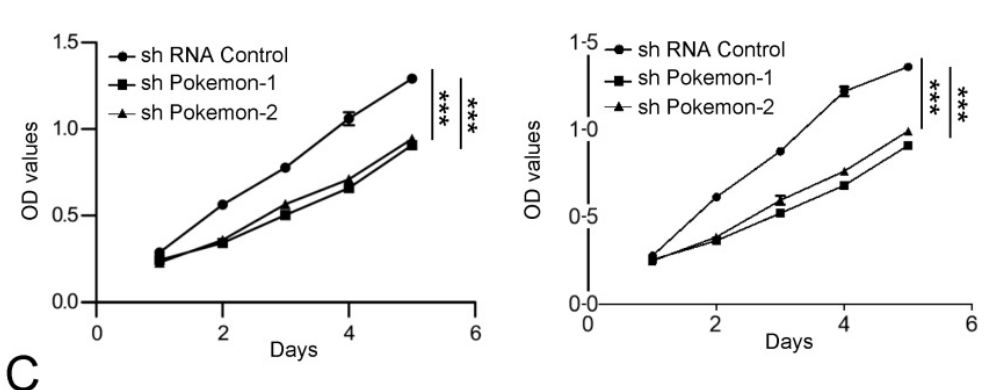
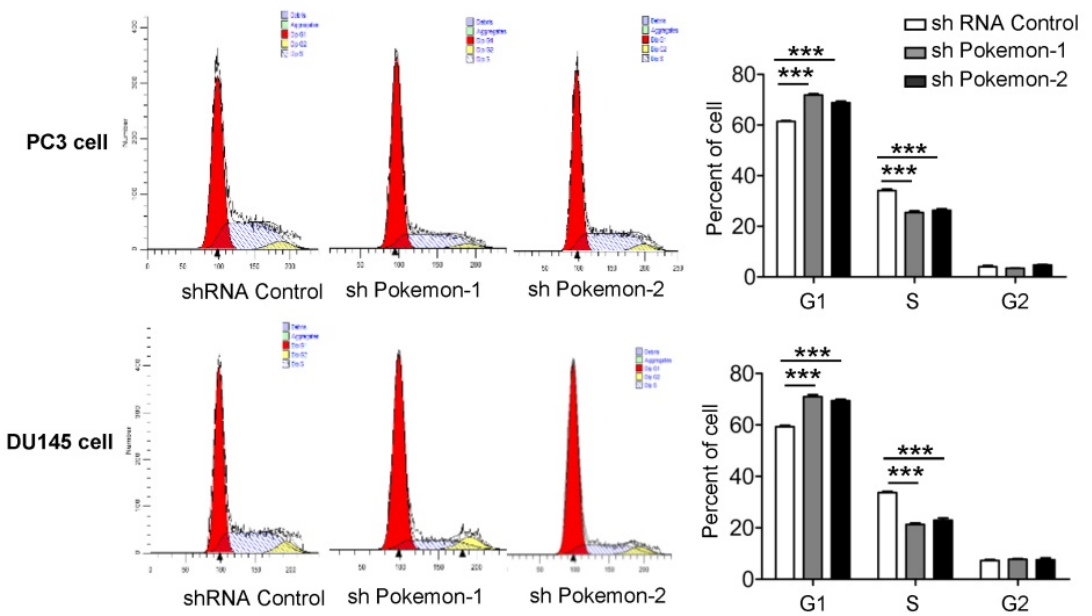

PC3 cell
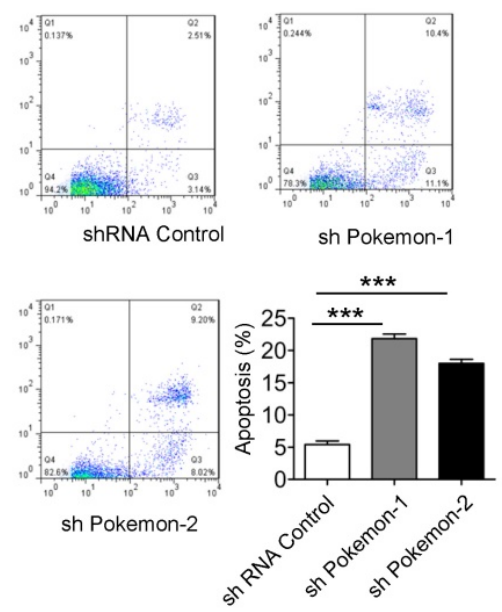

DU145 cell
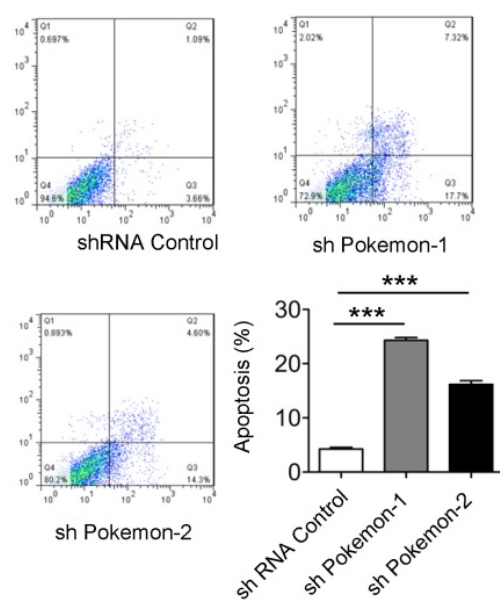

Figure 2. Analysis of Pokemon knockdown effects on cell proliferation and apoptosis. (A) mRNA and protein expression of Pokemon in PC 3 and DU145 cells after its knockdown by two different short hairpin RNA sequences (shRNAs). Control shRNA served as a control. (B) Analysis of viable cells (cell proliferation) at indicated time points in PC3 and DU145 cells after control and pokemon knockdown. (C) Cell cycle analysis after control and pokemon knockdown. (D) Apoptosis analysis by Annexin V/PI staining in PC3 and DU145 cells after control and pokemon knockdown. **p $<0.05$, *** $\mathrm{p}<0.01$, mean \pm SEM. 
C

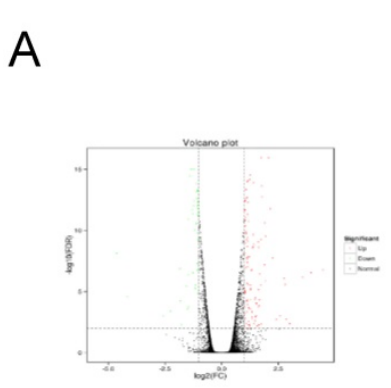

Differential expression of volcanic map
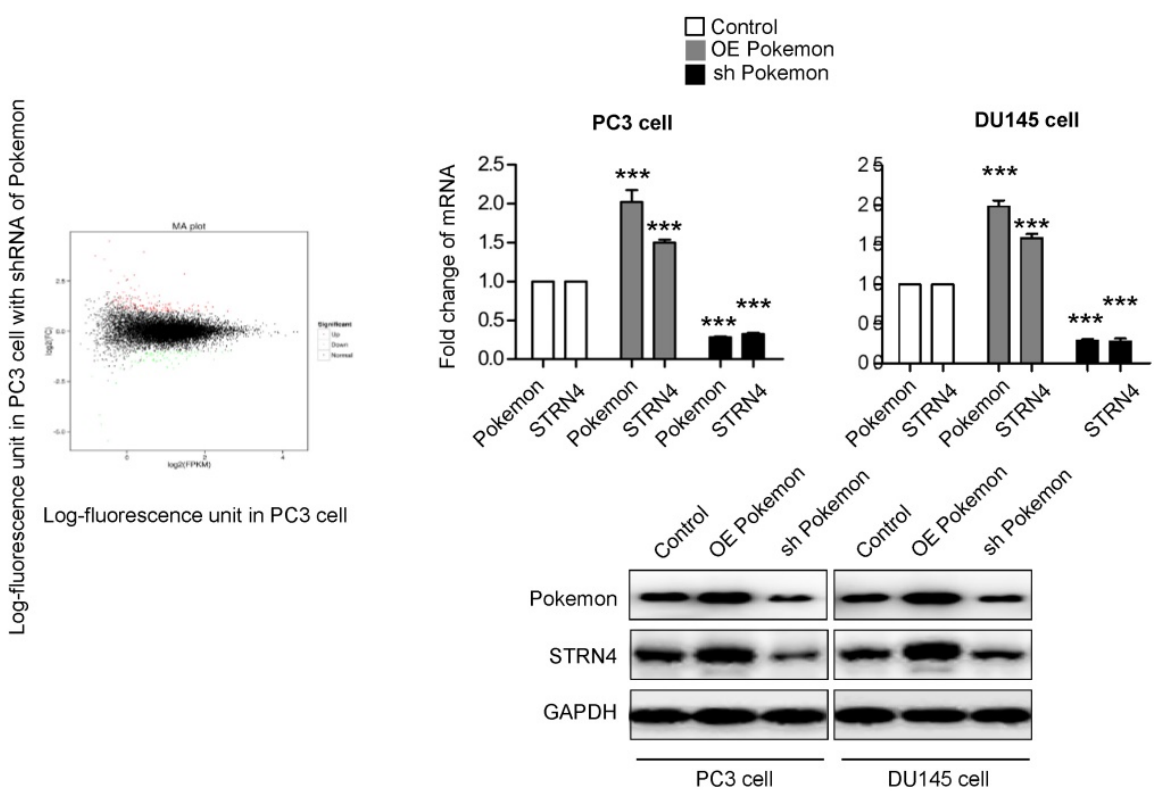

B

\begin{tabular}{|c|c|c|c|c|c|c|}
\hline Cell cycle & Apoptosis & $\begin{array}{c}\text { Transcription } \\
\text { Regulation }\end{array}$ & $\begin{array}{l}\text { Cell growth } \\
\text { Diffrentiation }\end{array}$ & $\begin{array}{c}\text { Signal } \\
\text { Transduction }\end{array}$ & $\begin{array}{l}\text { Biosynthesis } \\
\text { Metabolism }\end{array}$ & $\begin{array}{l}\text { Transport } \\
\text { ionchannel }\end{array}$ \\
\hline $\begin{array}{l}\text { STRN4 } \\
\text { CCND1 } \\
\text { ANO8 } \\
\text { CENPB } \\
\text { CASP7 }\end{array}$ & $\begin{array}{l}\text { TRIB3 } \\
\text { NGFR } \\
\text { RHOB } \\
\text { BCLAF1 }\end{array}$ & $\begin{array}{l}\text { DUSP1 } \\
\text { CARS } \\
\text { TRIB3 } \\
\text { RREB1 }\end{array}$ & $\begin{array}{l}\text { SUDS3 } \\
\text { CCNF } \\
\text { SLC37A2 } \\
\text { HAS2 }\end{array}$ & $\begin{array}{l}\text { EPHA1 } \\
\text { NPR3 } \\
\text { NOTCH3 } \\
\text { MAP4K4 } \\
\text { FHL3 } \\
\text { MKNK2 } \\
\text { FGF13 } \\
\text { SEMA6B } \\
\text { TRIB3 } \\
\text { RASGRP3 } \\
\text { NCAM2 } \\
\text { PLXNC1 } \\
\text { CIT } \\
\text { ACHE } \\
\text { PAK4 }\end{array}$ & $\begin{array}{l}\text { CYB5B } \\
\text { AOX1 } \\
\text { PRPS2 } \\
\text { GANAB } \\
\text { GUK1 } \\
\text { ALDH3A1 } \\
\text { LVRN } \\
\text { CYP2J2 }\end{array}$ & $\begin{array}{l}\text { SLC40A1 } \\
\text { KCND1 } \\
\text { KCNT2 } \\
\text { MT1M } \\
\text { NOX5 }\end{array}$ \\
\hline
\end{tabular}

Figure 3. Identification of the downstream target genes of Pokemon, and further validation of STRN4 as a target gene. (A) Scatterplots showing differential expression profile of Pokemon target genes in prostate cancer cells. (B) Pathway classification of identified target genes. (C) qPCR and western blot validation of STRN4 as the top target gene in PC3 and DU145 cells either overexpressing Pokemon or its knockdown. **p $<0.05$, ***p $<0.01$, mean \pm SEM

\section{Pokemon binds to STRN4 promoter and directly regulates its expression}

In an effort, to understand the molecular mechanism of Pokemon regulating STRN4 expression, we performed promoter motif analyses. Our results indicated that GGCGACCACCGA sequence located in STRN4 promoter can be recognized by Pokemon. To further verify this, we cloned the promoter region of STRN4 into a vector having downstream luciferase reporter, and transfected it into PC3 cancer cells (Figure 7A). Interestingly, it was observed that co-transfection of Pokemon with the reported vector, greatly enhanced luciferase activity in PC3 cells, while its knockdown significantly reduced the reporter activity (Figure 7B). This indicated that Pokemon is a positive regulator of STRN4 promoter. Furthermore, the interaction between Pokemon and STRN4 promoter was also confirmed by ChIP assay. As seen in Figure 7C, the promoter fragment of STRN4 was only amplified by polymerase chain reaction, when chromatin was precipitated with anti-Pokemon antibody, but not with nonspecific immunoglobulin G. Thus, it became evident that Pokemon regulated STRN4 expression through directly binding to its promoter region. 

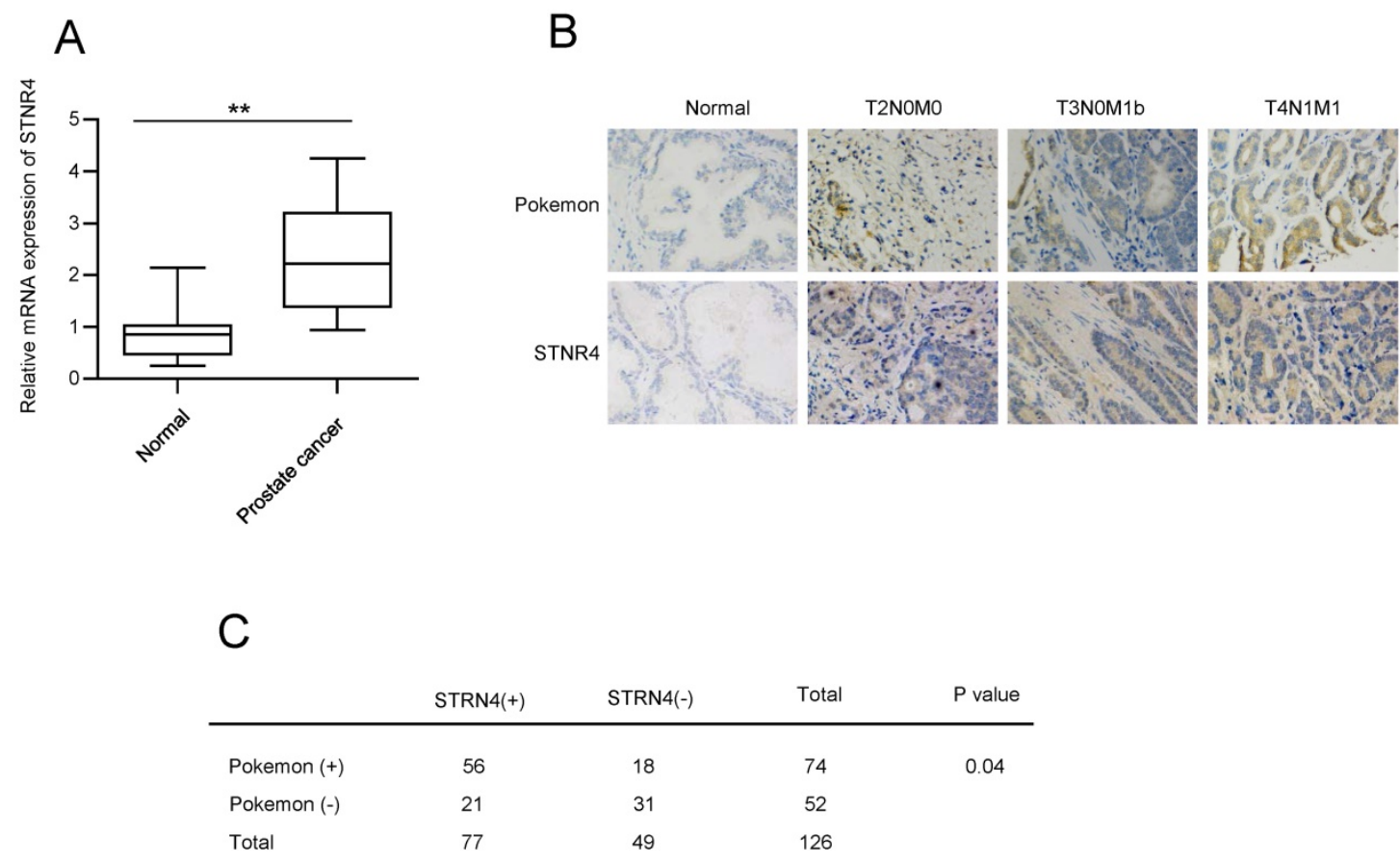

Figure 4. Analyzing the correlation between Pokemon and STRN4 expression. (A) qRTPCR analysis of the STRN4 expression in normal and prostate cancer tissue samples. (B) Parallel IHC analysis of Pokemon and STRN4 expression in prostate cancer tissue samples. (C) Overall summary of the Pokemon and STRN4 expression in 126 prostate cancer bio-specimens based on IHC analysis. ${ }^{* *} \mathrm{p}<0.05$, ${ }^{* * *} \mathrm{p}<0.01$, mean \pm SEM.

\section{Pokemon and STRN4 ablation suppresses xenograft prostate cancer cell growth.}

Finally, we also investigated the impact of Pokemon and STRN4 on xenograft growth of prostate cancer cells. PC3 cells with control (shControl), Pokemon (shPokemon) and STRN4 (shSTRN4) knockdown by respective shRNAs were injected subcutaneously into nude mice (6 injection sites per cell line), and tumor formation/growth was monitored over a three week time (Figure 8A). All injection sites formed tumors. After three weeks of cell injection, the mice were killed to measure tumor volumes. The average tumor volumes in shPokemon and shSTRN4 groups were significantly less in comparison to shControl cell group (Figure 8B) Moreover, a concomitant expression pattern was observed for STRN4 and Pokemon in knockdown cells (Figure 8C), which is consist with our in vitro study.

\section{Discussion}

Due to the lack of studies evaluating the role of Pokemon in prostate cancer, we in our current study have tried to assess its role and molecular mechanism in prostate cancer progression. Our results demonstrated that Pokemon is highly overexpressed in human prostate cancer tissue samples, in comparison to normal tissue. Furthermore, our study also revealed that pokemon knockdown in prostate cancer cells not only inhibited in vitro cell proliferation, but also suppressed xenograft growth. In addition, its knockdown suppressed cell cycle progression, and promoted cell apoptosis. In terms of molecular mechanism, we identified that pokemon effects on prostate cancer cell proliferation and apoptosis were mediated through its downstream target, STRN4. It was observed to directly bind to the promoter region of STRN4, and regulates its expression (Figure 9).

Multiple studies have shown that Pokemon overexpression promoted tumorigenesis, and acted as upstream of various tumor suppressing genes [19]. For instance, it has been shown that Pokemon promotes cell transformation by acting as a transcriptional repressor of tumor suppressor ARF/p53 pathway[20]. In addition, it has also been observed to interfere with GC box recognition by Sp1, which subsequently lead to the repression of ADH5/FDH transcription[21]. In our study, using illumina transcriptome sequencing, we showed that Pokemon regulates the expression of many genes, primarily involved in apoptosis, cell cycle, differentiation and biosynthesis. Moreover, we also identified that Pokemon can bind to GGCGACCACCGA sequence in the promoter region of STRN4 gene, and can directly induce its expression. This novel observation established a new signal pathway, by which Pokemon can not only act as a transcriptional repressor, but also can behave as transcriptional activator, to mediate oncogenesis. Even though, we indicated about the direct role of Pokemon in inducing STRN4 expression, 
we still advocate for additional studies to explore if any other downstream target of Pokemon is important for the regulation of STRN4 expression.

Earlier studies have indicated about the contribution of STRN4 in regulating proliferation, invasion and migration of various cancer cells, like lung, gastric, pancreatic, breast and colorectal [22, 23]. However, there is a lack of information about the signal pathway involved in mediating STRN4 contribution to tumor progression. Here in our study, by analyzing 126 prostate patient tumor biopsies, we demonstrated a strong correlation between STRN4 and Pokemon expression. Additional rescue studies did indicate that Pokemon acts upstream of STRN4, and can regulate its expression in prostate cancer. Overall, our study convincingly showed that Pokemon and STRN4 cooperate to drive prostate cancer cells proliferation and tumor progression, and thereby highlighting the potential role of Pokemon as a prognostic marker and therapeutic target for the treatment of prostate cancer.

A

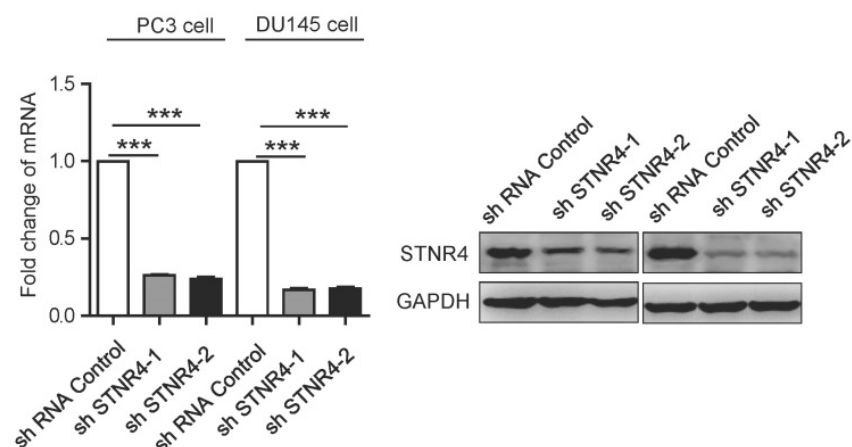

B

PC3 cell

DU145 cell
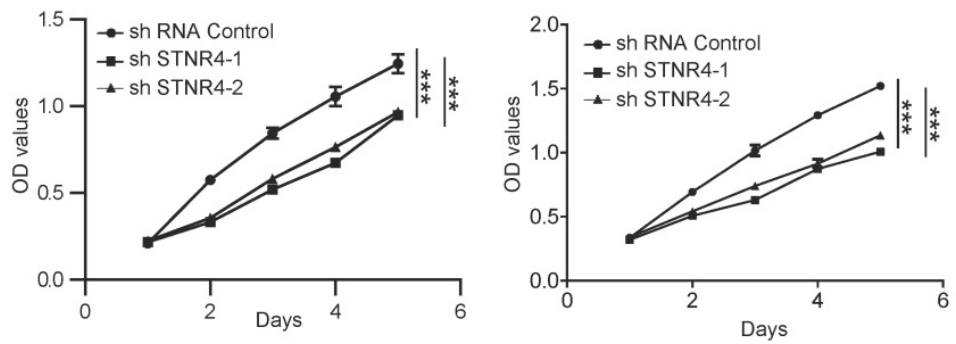

$\mathrm{D}$

$c$
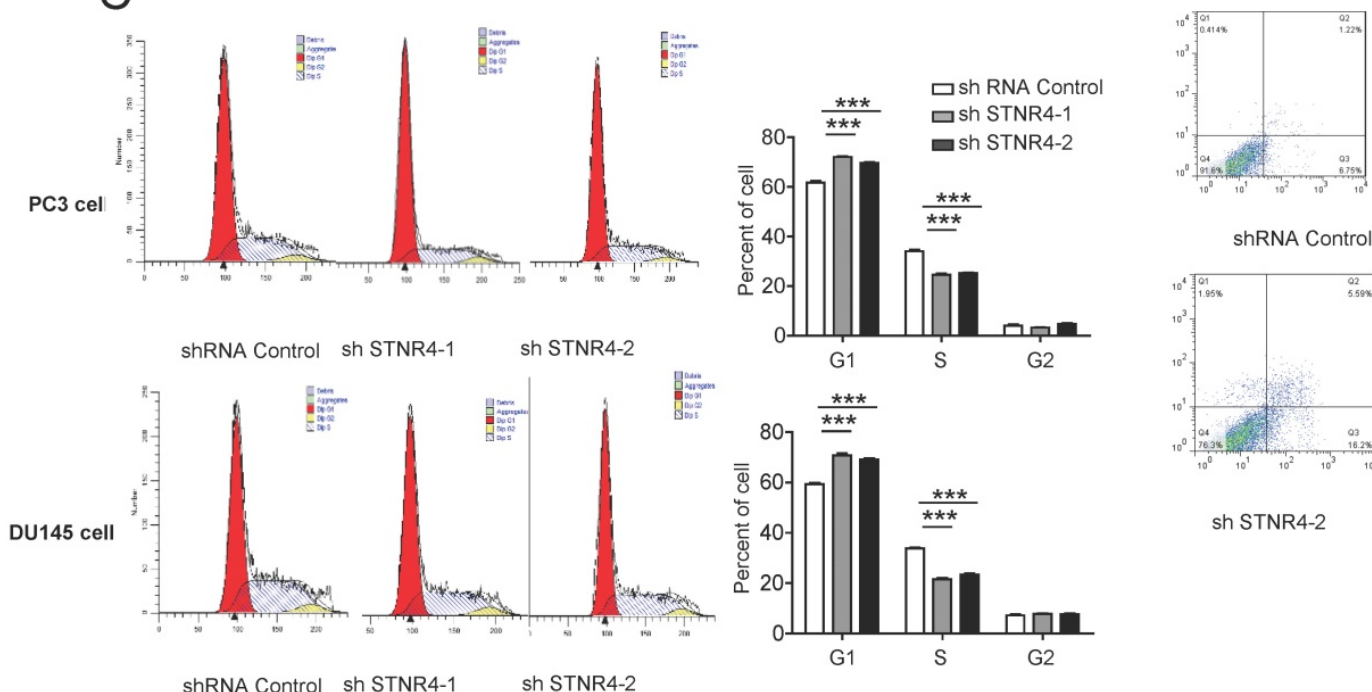

DU145 cell
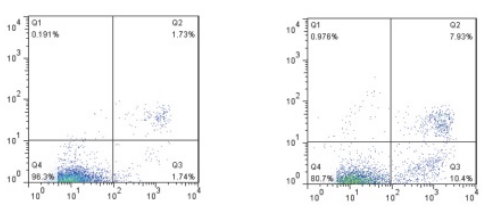

shRNA Contro

sh STNR4-1
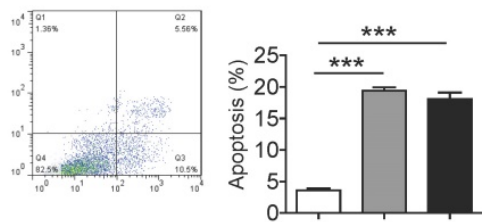

sh STNR4-2

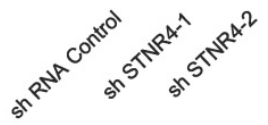

shRNA Control
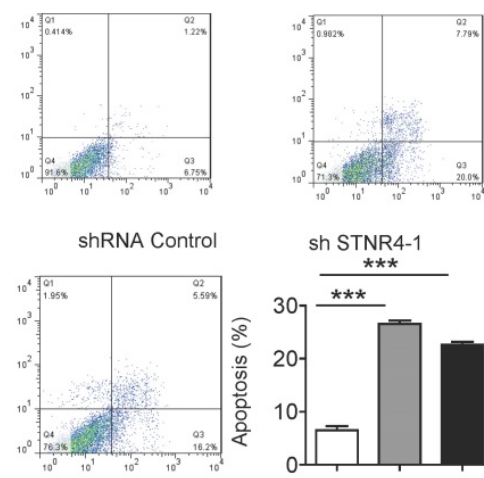

sh STNR4-1

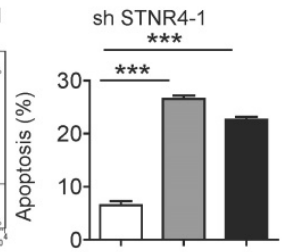

sh STNR4-2
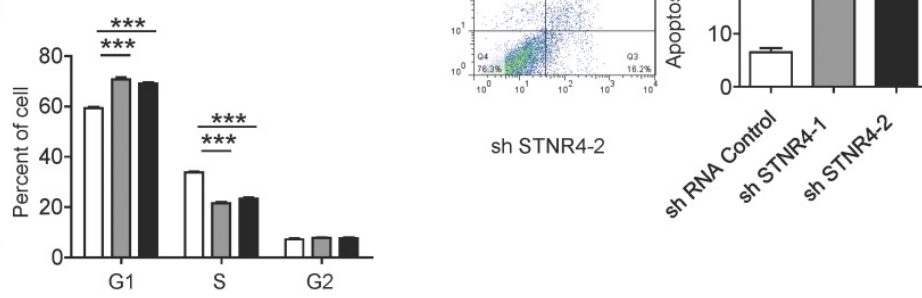

\section{5}

Figure 5. Analysis of the STRN4 knockdown effects on cell proliferation and apoptosis. After control and STRN4 knockdown by short hairpin RNAs (shRNAs) in
PC3 and DU145 cells, (A) qRTPCR and western blotting analysis of STRN4 expression; (B) analysis of the viable cells at indicated time points; (C) Cell cycle analysis; and (D) analysis of apoptosis by Annexin V/PI staining in cancer cells. ${ }^{* *} \mathrm{p}<0.05$, *** $<0.01$, mean $\pm \mathrm{SEM}$. 
A
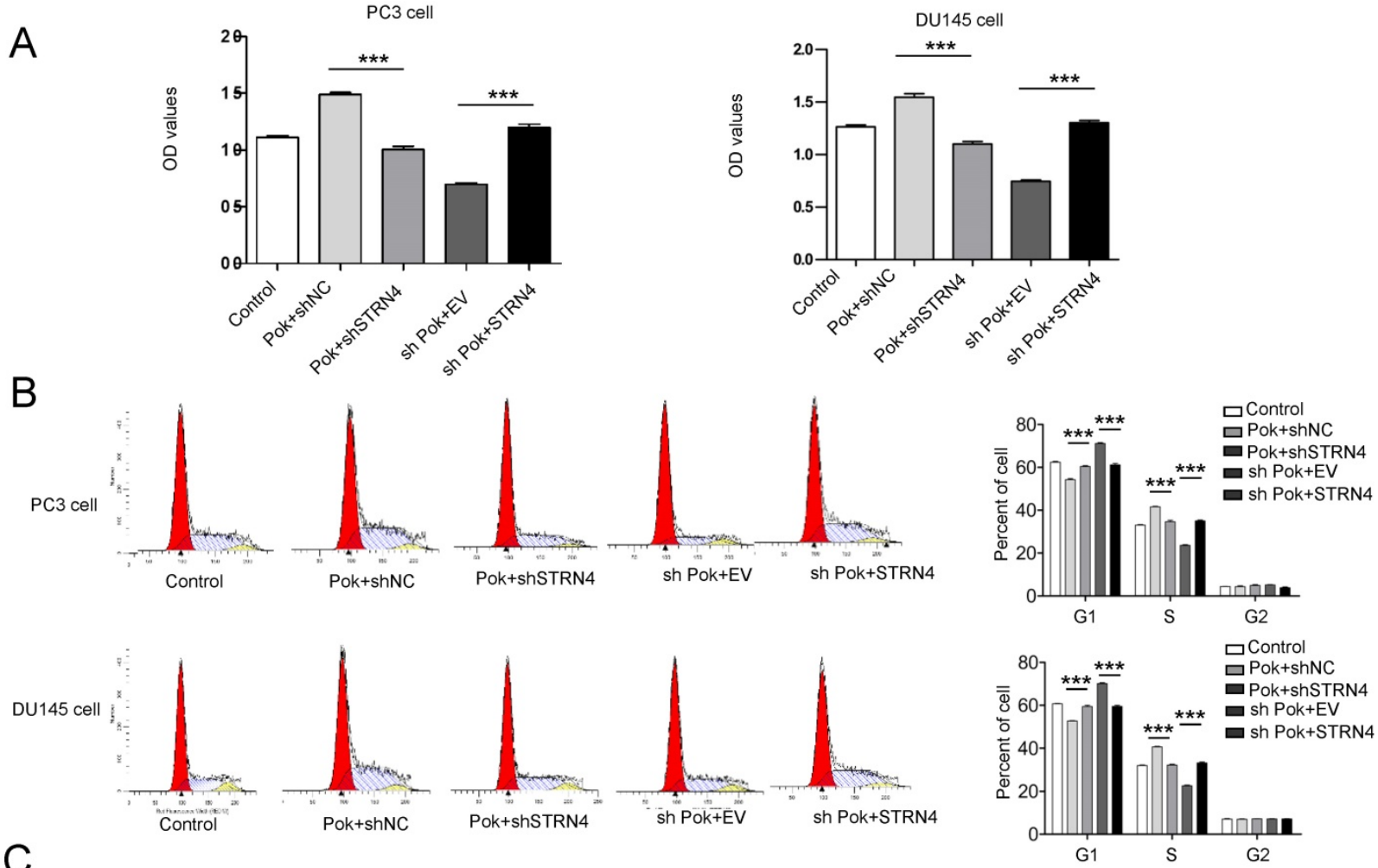

C

PC3 cell
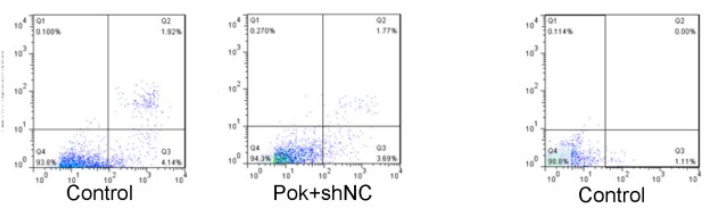

DU145 cell
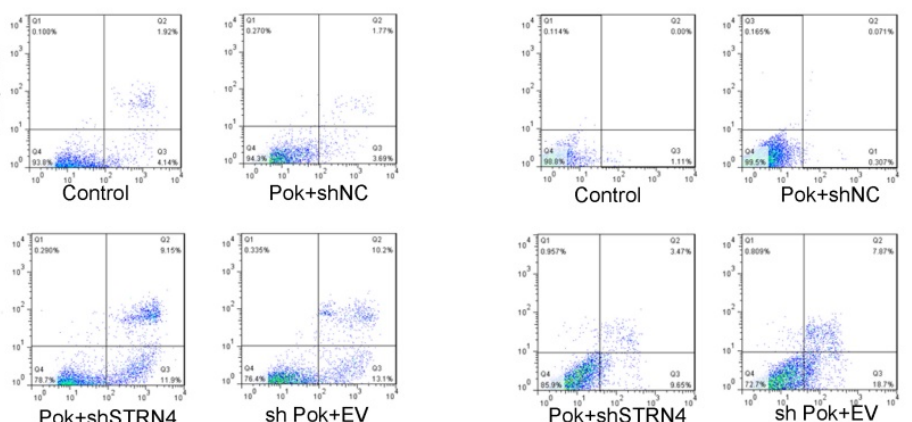

D
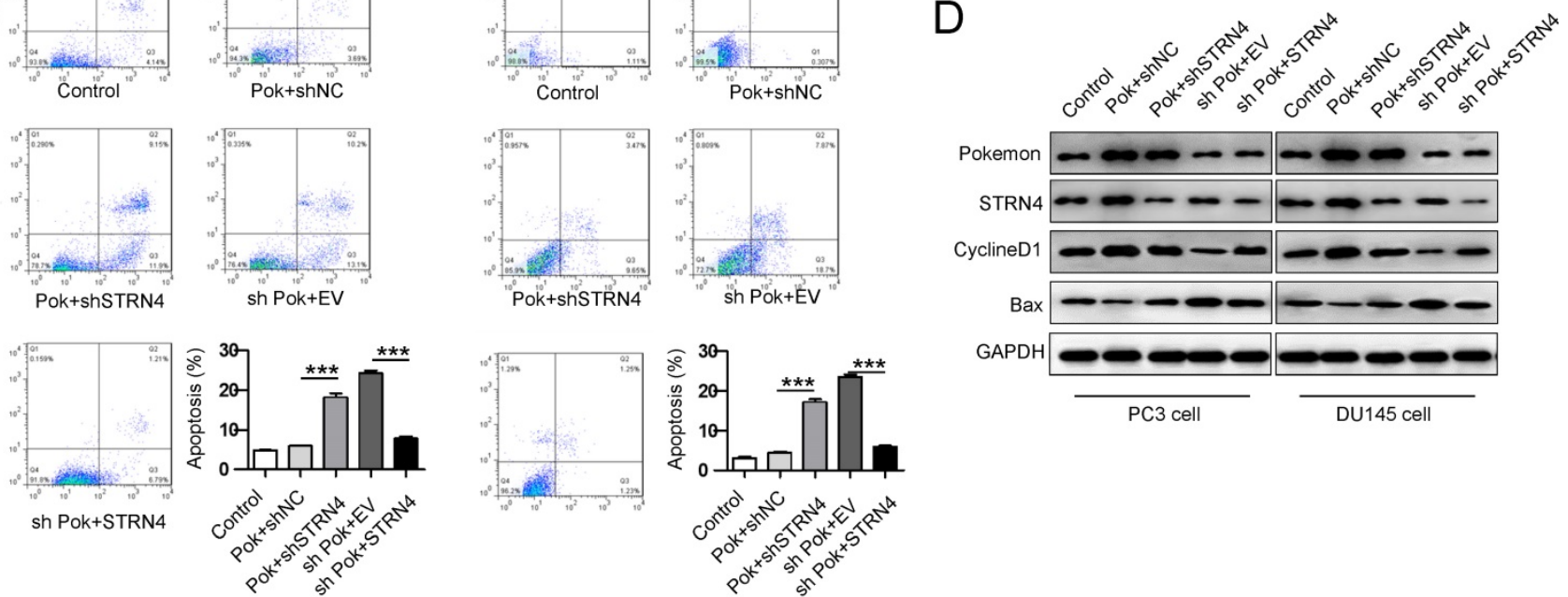

Figure 6. Effect of the parallel manipulation of Pokemon and STRN4 expression on prostate cancer cells proliferation, cell cycle and apoptosis. After PC3 and DU145 cells manipulation as follows, 1) Control transfection, 2) Pokmon overexpression + control shRNA, 3) Pokemon overexpression + STRN4 shRNA, 4) Pokemon shRNA + empty vector, and 5) Pokemon shRNA+ STRN4 overexpression, (A) cell proliferation analysis; (B) cell cycle progression analysis; (C) apoptosis analysis; and (D) western blot analysis of Pokemon, STRN4, cyclin DI, Bax and GAPDH protein expression. **p $<0.05$, **** $<0.01$, mean \pm SEM. 
A
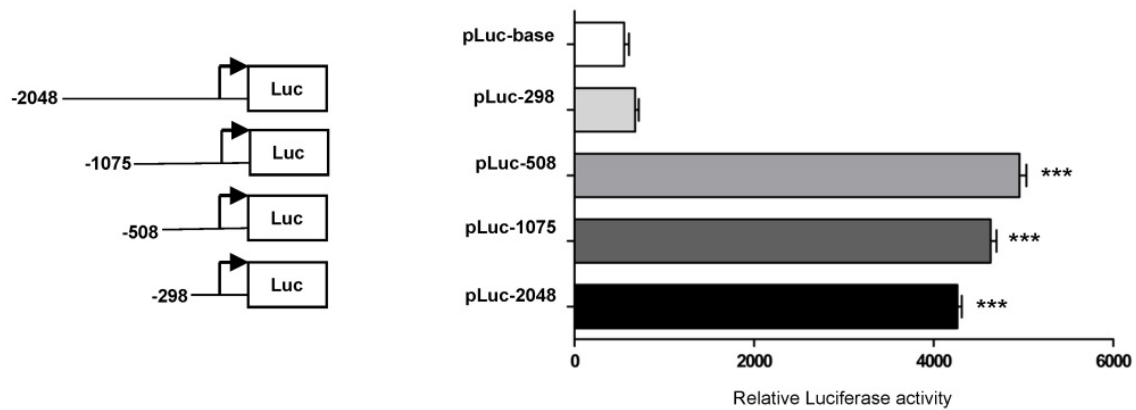

B
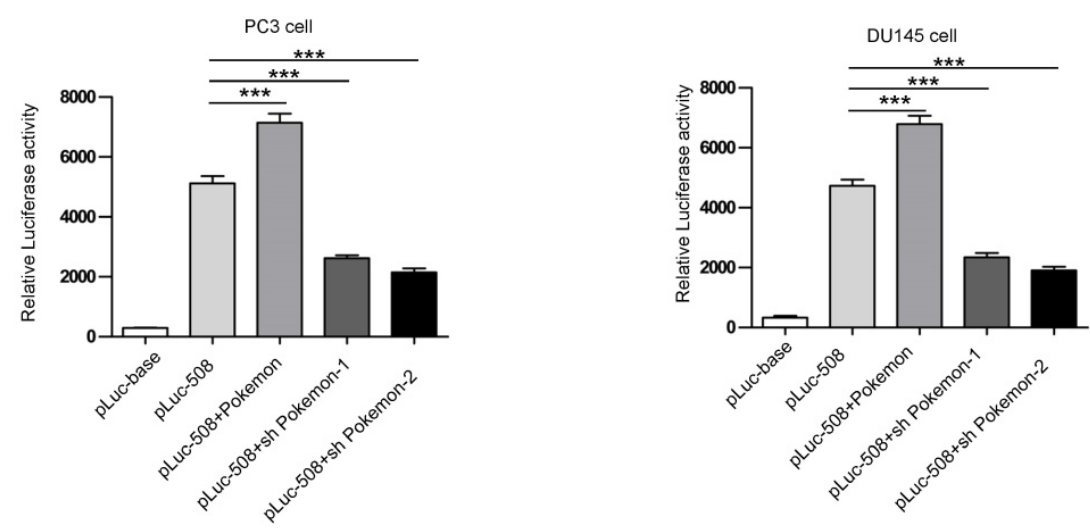

C

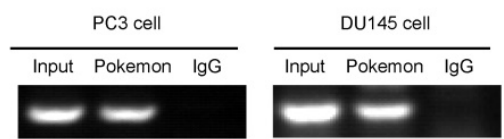

Figure 7. Analysis of Pokemon binding to STRN4 promoter. (A) Schematic representation of luciferase reporter plasmids with STRN4 promoter. (B) Analysis of STRN4 promoter activity in prostate cancer cells by luciferase assay, after co-transfection of pLuc-508 vector with Pokemon expressing cDNA or its shRNA. (C) CHIP assay to analyze Pokemon binding to STRN4 promoter in prostate cancer cells. $* * \mathrm{p}<0.05,{ }^{* * *} \mathrm{p}<0.01$, mean \pm SEM.

A

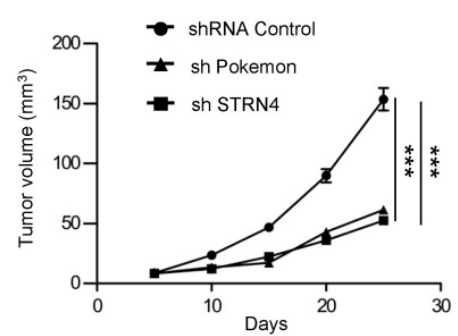

C

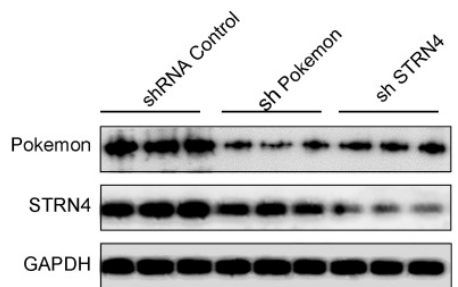

B
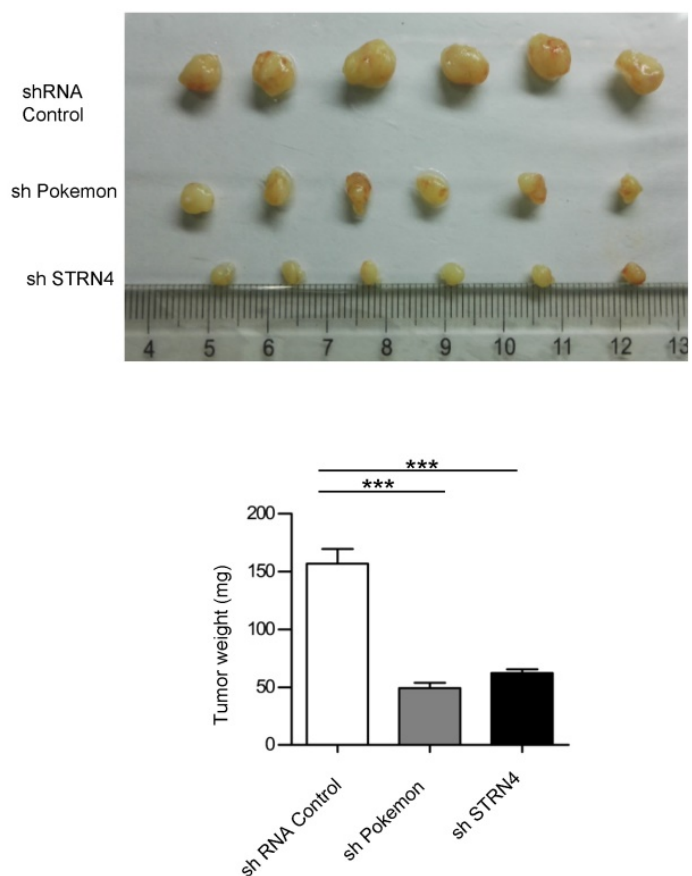

Figure 8. Effect of Pokemon and STRN4 knockdown on prostate cancer cell xenograft growth. (A) Average tumor volumes from six tumors obtained after subcutaneous injection of PC3 cells, with control (shControl), pokemon (shPokemon), and STRN4 (shSTRN4) knockdown, into nude mice. (B) Pictures depicting extracted tumors, and graph representing average tumor weight of six tumors in each group, after three weeks of cancer cell injection. (C) Western blotting analysis of Pokemon and STRN4 expression of extracted tumors. **p $<0.05$, ***p $<0.01$, mean \pm SEM. 


\section{Prostate cancer cells}

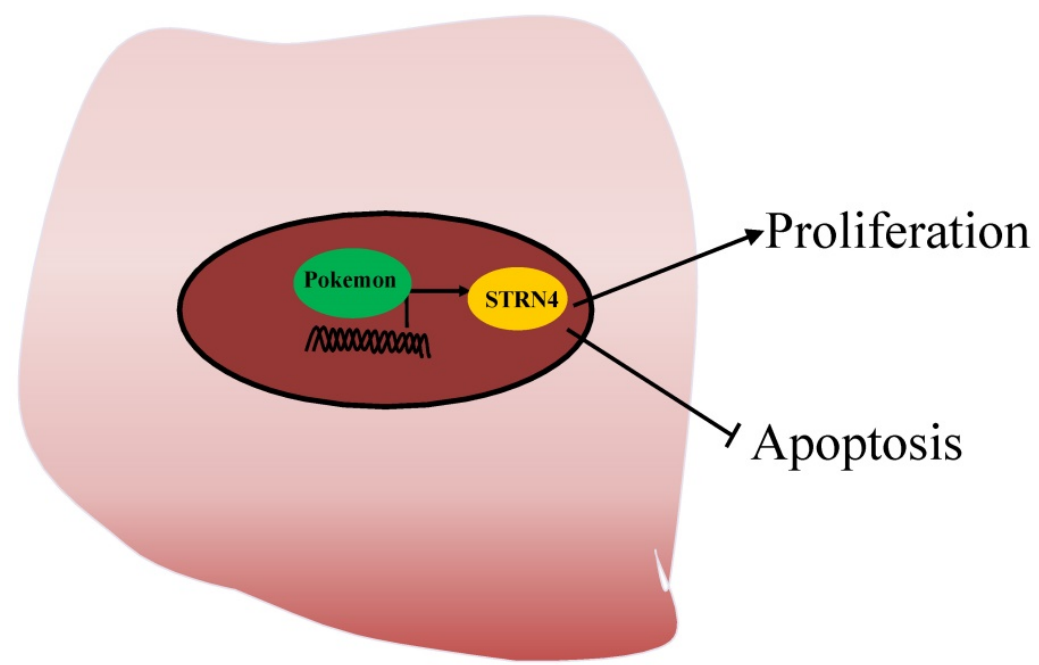

Figure 9. Working model of Pokemon-mediated prostate tumor progression. Pokemon directly binds to the promoter of STRN4, regulates its expression, which in turn promotes prostate cancer cell proliferation and suppress cancer cells apoptosis.

\section{Abbreviations}

CHIP: Chromatin immunoprecipitation; FBS: fetal bovine serum; GAPDH: glyceraldehyde 3-phosphate dehydrogenase; LRF: leukemia/lymphomarelated factor; PBS: phosphate-buffered saline; PI: propidium iodide; STRN4: striatin 4 .

\section{Ethical approval}

All applicable international, national, and/or institutional guidelines were followed for the care and use of animals. All human specimen studies were conducted in accordance with the ethical standards of the institutional and/or national research committee, along with 1964 Helsinki declaration guidelines.

\section{Informed consent}

All individual participants provided written informed consent to include in the study.

\section{Competing Interests}

The authors have declared that no competing interest exists.

\section{References}

1. Maeda T, RM Hobbs, T Merghoub,I Guernah. Role of the proto-oncogene Pokemon in cellular transformation and ARF repression. Nature.2005;433: 278

2. Aggarwal A, WJ Hunter, 3rd, H Aggarwal, ED Silva, MS Davey, RF Murphy, et al. Expression of leukemia/lymphoma-related factor (LRF/POKEMON) in human breast carcinoma and other cancers. Exp Mol Pathol.2010;89: 140-8

3. Rovin RA,R Winn. Pokemon expression in malignant glioma: an application of bioinformatics methods. Neurosurg Focus.2005;19: E8

4. Zhao ZH, SF Wang, L Yu, J Wang, H Chang, WL Yan, et al. Overexpression of Pokemon in non-small cell lung cancer and foreshowing tumor biological behavior as well as clinical results. Lung Cancer.2008;62: 113-9

5. Aggarwal H, A Aggarwal, WJ Hunter, 3rd, P Yohannes, AU Khan, and DK Agrawal. Expression of leukemia/lymphoma related factor (LRF/Pokemon) in human benign prostate hyperplasia and prostate cancer. Exp Mol Pathol.2011;90: 226-30

6. Maeda T, RM Hobbs, T Merghoub, I Guernah, A Zelent, C Cordon-Cardo, et al. Role of the proto-oncogene Pokemon in cellular transformation and ARF repression. Nature.2005;433: 278-285

7. Lee D-K, D Suh, HJ Edenberg,M-W Hur. POZ domain transcription factor, FBI-1, represses transcription of ADH5/FDH by interacting with the zinc finger and interfering with DNA binding activity of Sp1. Journal of Biological Chemistry.2002;277: 26761-26768

8. Cui J, X Meng, X Gao,G Tan. Curcumin decreases the expression of Pokemon by suppressing the binding activity of the Sp1 protein in human lung cancer cells. Molecular biology reports.2010;37: 1627-1632

9. Lee DK, JE Kang, HJ Park, MH Kim, TH Yim, JM Kim, et al. FBI-1 enhances transcription of the nuclear factor-kappaB (NF-kappaB)-responsive E-selectin gene by nuclear localization of the p65 subunit of NF-kappaB. J Biol Chem.2005;280: 27783-91

10. Jiang L, MK Siu, OG Wong, KF Tam, EW Lam, HY Ngan, et al. Overexpression of proto-oncogene FBI-1 activates membrane type 1-matrix metalloproteinase in association with adverse outcome in ovarian cancers. Mol Cancer.2010;9: 318

11. Laudes M, R Bilkovski, F Oberhauser, A Droste, M Gomolka, U Leeser, et al. Transcription factor FBI-1 acts as a dual regulator in adipogenesis by coordinated regulation of cyclin-A and E2F-4. J Mol Med (Berl).2008;86: 597-608

12. Laudes M, R Bilkovski, F Oberhauser, A Droste, M Gomolka, U Leeser, et al. Transcription factor FBI-1 acts as a dual regulator in adipogenesis by coordinated regulation of cyclin-A and E2F-4. Journal of Molecular Medicine. 2008;86: 597-608

13. Castets F, M Bartoli, J Barnier, G Baillat, P Salin, A Moqrich, et al. A novel calmodulin-binding protein, belonging to the WD-repeat family, is localized in dendrites of a subset of CNS neurons. The Journal of cell biology.1996;134: 1051-1062

14. Muro Y, EK Chan, G Landberg,EM Tan. A Cell-Cycle Nuclear Autoantigen Containing Wd-40 Motifs Expressed Mainly in S and G2-Phase Cells. Biochemical and biophysical research communications.1995;207: 1029-1037

15. Wong M, T Hyodo, E Asano, K Funasaka, R Miyahara, Y Hirooka, et al. Silencing of STRN4 suppresses the malignant characteristics of cancer cells. Cancer Sci.2014;105: 1526-32

16. Haque A, MA Rahman, JR Fuchs, ZG Chen, FR Khuri, DM Shin, et al. FLLL12 induces apoptosis in lung cancer cells through a p53/p73-independent but death receptor 5-dependent pathway. Cancer letters.2015;363: 166-175

17. Sartini D, L Lo Muzio, S Morganti, V Pozzi, G Di Ruscio, R Rocchetti, et al. Pokemon proto-oncogene in oral cancer: potential role in the early phase of tumorigenesis. Oral diseases.2015;21: 462-469

18. Rheinbay E, P Parasuraman, J Grimsby, G Tiao, JM Engreitz, J Kim, et al Recurrent and functional regulatory mutations in breast cancer. Nature.2017;547: 55-60

19. Maeda T, RM Hobbs,PP Pandolfi. The transcription factor Pokemon: a new key player in cancer pathogenesis. Cancer Research.2005;65: 8575-8578

20. Taneja P, D Maglic, F Kai, T Sugiyama, RD Kendig, DP Frazier, et al. Critical roles of DMP1 in human epidermal growth factor receptor 2/neu-Arf-p53 signaling and breast cancer development. Cancer Res.2010;70: 9084-94 
21. Roh HE, MN Lee, BN Jeon, WI Choi, YJ Kim, MY Yu, et al. Regulation of pokemon 1 activity by sumoylation. Cell Physiol Biochem.2007;20: 167-80

22. Madsen CD, S Hooper, M Tozluoglu, A Bruckbauer, G Fletcher, JT Erler, et al.

STRIPAK components determine mode of cancer cell migration and metastasis. Nature cell biology.2015;17: 68

23. Wong M, T Hyodo, E Asano, K Funasaka, R Miyahara, Y Hirooka, et al. Silencing of STRN4 suppresses the malignant characteristics of cancer cells. Cancer science.2014;105: 1526-1532 